DOI: https://doi.org/10.11144/Javeriana.upsy16-2.ceav

\title{
Contenido experiencial y aspectos valorativos asociados a la envidia benigna y maligna en estudiantes universitarios de Córdoba, Argentina*
}

\section{Experiential Content and Appraisal Pattern associated with Benign and Malicious Envy in University Students of Cordoba, Argentina}

\author{
Débora Jeanette Mola ${ }^{a}$ \\ Universidad Nacional de Córdoba, Argentina \\ ORCID: http://orcid.org/0000-0002-7810-2424 \\ Juan Carlos Godoy \\ Universidad Nacional de Córdoba, Argentina \\ Cecilia Reyna \\ Universidad Nacional de Córdoba, Argentina
}

a Autora de correspondencia. Correo electrónico: deboramola@gmail.com

Para citar este artículo: Mola, D., Reyna, C., \& Godoy, J. (2017). Contenido Experiencial y Aspectos Valorativos asociados a la Envidia Benigna y Maligna en Estudiantes Universitarios de Córdoba, Argentina. Universitas Psychologica 16(2), 1-12.

https://doi.org/10.11144/Javeriana.upsy16-2.ceav

\section{RESUMEN}

La envidia ha sido considerada una experiencia desagradable caracterizada por el anhelo de que la persona envidiada se vea perjudicada. Estudios recientes realizados en otros países evidencian la existencia de una envidia cualitativamente distinta, denominada benigna. El envidioso benigno valora la situación como inspiradora y se siente motivado para mejorar. Así, en esta investigación se evaluó el contenido experiencial y el patrón valorativo de la envidia benigna y maligna en estudiantes universitarios de Córdoba, Argentina. Se utilizó el método de reconstrucción del día para recabar información sobre envidia. Los resultados evidenciaron en el contexto local la existencia de dos tipos de envidia distinguidas por los aspectos valorativos de merecimiento, poder propio y estado situacional. Además, se observó que la envidia benigna desencadena inspiración y motiva a felicitar al envidiado.

Palabras clave

envidia benigna; envidia maligna; contenido experiencial; patrones valorativos

\section{ABSTRACT}

Envy has been regarded an unpleasant experience characterized by the wish that the envied person is impaired. Recent studies conducted in other countries evidence the existence of a qualitatively different envy, named benign. People who experience benign envy assess the situation as inspiring and are motivated to improve. Thus, in this research we evaluated the experiential content and appraisal patterns of benign and malicious envy in a sample of undergraduate students from Cordoba, Argentina. The day reconstruction method was used to recover information about envy. Results evidenced that two types of envy are experienced at the local context, which are distinguished considering the following items: deservingness, own power and situational state. Furthermore, it was observed that the experience of benign envy triggers inspiration and motivates congratulate to the envied person.

Keywords

benign envy; malicious envy; experiential content; appraisals patterns 


\section{Introducción}

Frecuentemente, las personas que nos rodean tienen mejor desempeño que nosotros, tu compañero de equipo puede ser mejor en básquet, o un colega puede recibir un prestigioso premio que anhelabas obtener. A menudo, estas comparaciones ascendentes conducen a la experiencia emocional de la envidia. Esta emoción surge cuando una persona carece de un atributo deseado y se caracteriza por el anhelo de que la persona que lo posee lo pierda o que de alguna manera se vea perjudicada (Parrott $\&$ Smith, 1993). La envidia ha sido considerada una experiencia hedónicamente desagradable (Smith, Parrott, Diener, Hoyle, \& Kim, 1999). De hecho, estudios empíricos recientes comprobaron que la mayoría de las personas parecen ser capaces de sentirla en diferentes culturas (Van de Ven, Zeelenberg, \& Pieters, 2009). Como plantean Chóliz y Gómez (2002), la envidia es una emoción social o secundaria dado que surge de un contexto social específico y se trata de una experiencia que adquiere sentido en relación con los demás. Es decir, es una emoción que se produce por comparación con los demás, y tanto las valoraciones cognitivas como las conductas manifiestas están dirigidas hacia otra persona que dispone de lo que carece el envidioso (Smith et al., 1999). A su vez, algunos teóricos han acordado que la condición mínima para que se desencadene la envidia es la comparación social ascendente (Chóliz \& Gómez, 2002; Parrott \& Smith, 1993; Smith et al., 1999). En este sentido, estudios empíricos que indagan las respuestas emocionales y neuronales de la comparación social ascendente evidencian que la disminución en la actividad del estriado ventral y el incremento de dicha actividad en la corteza cingulada anterior derecha y la corteza prefrontal ventromedial se asocian al desencadenamiento de la envidia (Dvash, Gilam, Ben-Ze'ev, Hendler, \& Shamay-Tsoory, 2010; Jankowski \& Takahashi, 2014; ShamayTsoory, Tibi-Elhanany, \& Aharon-Peretz, 2007). Además, Smith et al. (1999) sostienen que esta emoción se caracteriza a partir de dos componentes afectivos principales: sentimientos de inferioridad y sentimientos de mala voluntad. Los primeros se desencadenan a partir de la comparación social ascendente, la cual provoca una disminución en la autoestima. En tanto que los segundos surgen cuando otra persona que es similar en la mayoría de los aspectos a la persona envidiosa, goza de una ventaja considerada inalterable (Parrott \& Smith, 1993, Smith et al., 1999). Asimismo, Parks, Rumble y Posey (2002) plantean que otra característica relevante de la envidia es el sentimiento de injustica. En concreto, los autores describen que la ventaja del otro puede ser percibida como justa o injusta dependiendo de si el envidiado merece o no dicha ventaja.

Si bien las investigaciones se han centrado tradicionalmente en el estudio de la envidia como una emoción homogénea, varios autores han propuesto la existencia de dos facetas o formas de envidia (Cohen-Charash, 2009; Foster, 1972; Smith \& Kim, 2007). Por un lado, una forma más positiva, donde la envidia es considerada como una fuerza motivadora que impulsa a la gente a trabajar más duro para conseguir lo que otros ya tienen (Foster, 1972) y, por otro lado, una forma más negativa, la cual ha sido indagada frecuentemente en las investigaciones empíricas (e.g., Hoelzl \& Loewenstein, 2005; Parks et al., 2002). No obstante, algunos autores sostienen que la distinción entre los tipos de envidia se debe exclusivamente a la presencia o ausencia de sentimientos hostiles, argumentando que solo la envidia que presenta este componente hostil debe considerarse envidia propiamente dicha (Smith \& Kim, 2007).

Además, investigaciones recientes evidencian la existencia de dos tipos de envidia: maligna y benigna ("malicious y benign" en la literatura en inglés) (Cohen-Charash, 2009; Lange \& Crusius, 2015; Van de Ven et al., 2009; Van de Ven, Zeelenberg, \& Pieters, 2010; Van de Ven, Zeelenberg, \& Pieters, 2012). En este sentido, considerando que las emociones tienen un función pragmática mediante la preparación y motivación para la acción (Zeelenberg \& Pieters, 2006) los dos tipos de envidia pueden desencadenar comportamientos diferentes, e.g., las personas 
envidiosas benignamente incrementaron su comportamiento pro-social mientras que los envidiosos malignamente no lo hicieron (Van de Ven et al. 2010). No obstante, es necesario destacar que los estudios desarrollados en este campo arrojan resultados contradictorios. Así, por ejemplo, Mola, Reyna y Godoy (2015) no encontraron diferencias estadísticamente significativas al examinar la influencia de la envidia benigna y maligna en la toma decisiones económicas.

Por otro lado, diferentes estudios señalan que los dos tipos de envidia se caracterizan por poseer contenidos experienciales distintos. Es decir, se diferencian en los sentimientos, los pensamientos, las tendencias de acción y las metas emo-motivacionales que componen la experiencia emocional de la envidia (Crusius \& Lange, 2014; Van de Ven et al. 2009). Así, Van de Ven et al. (2009) observaron que las personas que experimentaron envidia maligna sentían más frustración, percibían la ventaja como injusta y esperaban que el otro fracasara, a diferencia de las personas que experimentaban envidia benigna, donde la situación era experimentada como inspiradora y provocaba tendencias de acción centradas en el esfuerzo para mejorar. Además, reportaron que la experiencia de envidia benigna motivaba a la persona envidiosa a realizar mejoras sobre sí mismo (en el atributo deseado) para alcanzar la posición superior en la que se encontraba el envidiado. Mientras que en la experiencia de envidia maligna la motivación del envidioso estaba dirigida a tirar hacia abajo o a una posición inferior al envidiado (Van de Ven et al. 2009).

Adicionalmente, varios autores han propuesto que una persona envidiosa percibe la situación como inmerecida y escasamente controlable (Parks et al., 2002; Smith et al., 1999). De igual manera, Van de Ven et al. (2012) sostienen que los tipos de envidia se pueden distinguir a partir de los aspectos valorativos de merecimiento y potencial de control. En este sentido, se observó que los participantes que sintieron envidia maligna percibieron la situación como menos merecida que los participantes que sintieron envidia benigna, mientras que estas últimas personas valoraron la situación como más controlable que las primeras (Van de Ven et al., 2012).

Asimismo, Van de Ven (2009) ha sugerido indagar sobre el entitlement o derecho psicológico para comprender si tiene efectos diferenciales entre las personas envidiosas, dado que las personas que tienden a sentirse con más derecho que los otros pueden percibir la ventaja del otro como inmerecida $y$, por lo tanto, experimentar con mayor frecuencia envidia maligna que benigna. No obstante, vale señalar que el merecimiento (deservingness en la literatura en inglés) ha sido diferenciado del derecho psicológico (Feather, 2003; Van de Ven et al., 2012). El primero se relaciona con la estructura valorativa de las acciones y sus resultados, y brinda información sobre la emoción desencadenada (Feather, 2006; Feather $\&$ McKee, 2009). Mientras que el segundo ha sido conceptualizado como un sentido estable de que uno tiene más derecho que los otros, implica un estado intrapsíquico global y se refleja en conductas actuales o deseadas, es decir, que no refiere necesariamente a un derecho que surge de una situación específica (Campbell, Bonnacci, Shelton, Exline, \& Bushman, 2004). Además, Feather (2003) sostiene que el concepto de entitlement hace referencia a un marco externo de derechos, reglas y normas sociales. No obstante, Van de Ven (2009) sugiere la utilización complementaria de las medidas de envidia disposicional y entitlement para comprender los efectos diferenciales de la envidia entre las personas que se consideran merecedoras de la situación y aquellas que no.

Aunque la envidia ha sido considerada una emoción universal (Foster, 1972; Smith et al., 1999; Van de Ven et al., 2009), pueden manifestarse diferencias a nivel individual $\mathrm{y}$ cultural (Fischer \& Manstead, 2008). Por lo tanto, resulta de interés evaluar esta emoción en el contexto local, indagando las particulares expresiones de envidia benigna y maligna. $\mathrm{Si}$ bien en nuestro país se utiliza la etiqueta "envidia" para ambos tipos de envidia, en ocasiones se aprecian expresiones como "envidia de la buena" o "envidia sana" y "envidia 
de la mala" u otras expresiones similares, lo cual supone una diferenciación categórica entre las mismas. Así, esta investigación se propuso evaluar el contenido experiencial y el patrón valorativo asociados a los dos tipos de envidia en una muestra de estudiantes universitarios de Córdoba, Argentina.

\section{Método y Materiales}

\section{Participantes}

La muestra estuvo compuesta por 54 estudiantes universitarios con edades comprendidas entre los 18 y 25 años $(\mathrm{M}=$ 21.59, $\mathrm{DS}=2.11)$ de ambos sexos (41 (75.9\%) mujeres, 13 (24.1\%) varones) que cursaban en distintas facultades de la Universidad Nacional de Córdoba. La muestra fue seleccionada de manera auto-elegida, ya que

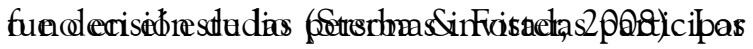
participantes recibieron información de forma escrita sobre las condiciones principales del estudio, donde se destacó la voluntariedad de su participación y la confidencialidad de la información brindada.

\section{Instrumentos}

Se utilizó la Dispositional Envy Scale (Smith et al., 1999), que es una medida de autoreporte que mide la tendencia a sentir envidia. La escala comprende 8 ítems con un formato de respuesta tipo Likert de 5 puntos $(1=$ muy en desacuerdo, $5=$ muy de acuerdo). Estudios originales de la escala han evidenciado a través de métodos exploratorios y confirmatorios su unidimensionalidad, resultando también una medida con adecuadas propiedades de consistencia interna (alfa de Cronbach $=0.83$ a 0.86) y estabilidad (correlación test-retest de 0.80). En estudios locales previos conducidos por nuestro grupo de investigación se ha replicado la estructura unidimensional de la escala y se ha obtenido evidencia de adecuadas propiedades de confiabilidad (Mola, Saavedra, \& Reyna, 2014).
También se aplicó la Psychological Entitlement Scale (PES, Campbell et al., 2004), la cual evalúa el derecho psicológico como fenómeno intra-psíquico global. Esta escala está compuesta por 9 ítems con un formato de respuesta tipo Likert de 5 puntos $(1=$ muy en desacuerdo, $5=$ muy de acuerdo). Dicha escala ha sido examinada en el contexto local evidenciando propiedades psicométricas adecuadas (Mola, Saavedra, Reyna, \& Belaus, 2013). Los análisis factoriales exploratorios y confirmatorios evidenciaron que ocho de los nueve ítems que comprendía la escala original mostraron una estructura unifactorial, con adecuadas cargas factoriales y/o pesos de regresión. En cuanto a la consistencia interna, se observaron valores aceptables de coeficientes alfa de Cronbach (0.86 y 0.81 , respectivamente para las muestras).

Además, se utilizó el Método de reconstrucción del día (Kahneman, Krueger, Schkade, Schwarz, \& Stone, 2004) para la recolección de las experiencias de envidia. Los participantes indicaron si habían experimentado envidia durante el día, si la respuesta era afirmativa debían describir brevemente su experiencia y responder a una serie de preguntas que aluden a los siguientes aspectos: a) Intensidad de la experiencia de envidia, con una escala de respuesta tipo Likert de 9 puntos $(1=$ para nada intensa, 9= muy intensa) (Van de Ven et al., 2009); b) Facilidad para recordar la experiencia de envidia, con una escala de respuesta tipo Likert de 9 puntos $(1=$ muy difícil, $9=$ muy fácil) (Van de Ven et al., 2009); c) Contenido experiencial de la envidia, los participantes respondieron a ocho ítems sobre contenido experiencial, con una escala de respuesta tipo Likert de 3 puntos $(1=$ para nada o muy poco, $2=$ poco, 3 = mucho o muchísimo), para diferenciar entre envidia benigna y maligna (Roseman, Wiest, \& Swartz, 1994; Van de Ven et al., 2009). Algunos de los ítems fueron los siguientes: lo sentí placentero; me sentí inspirado/a por la persona a la que envidié y me esforcé más para lograr mis objetivos; y d) Aspectos valorativos de la envidia: los participantes respondieron a diez preguntas designadas para medir los aspectos valorativos. Se incluyeron siete dimensiones 
planteadas por Roseman, Antoniou y Jose (1996) y se añadió la valoración de merecimiento (Van de Ven et al., 2012) para diferenciar entre envidia benigna y maligna. Algunas de las valoraciones del evento consideradas fueron las siguientes: estado de la situación, estado motivacional y merecimiento (Roseman et al., 1996; Van de Ven et al., 2012). Los ítems empleados para recuperar los distintos aspectos de las experiencias de envidia fueron traducidos al castellano. En la sección de procedimiento se presenta con mayor detalle el análisis realizado.

\section{Procedimiento}

En primer lugar, se procedió a la traducción de los ítems originales que corresponden a las variables contenido experiencial, aspectos valorativos, intensidad, y facilidad mediante el procedimiento de traducción inversa (Vergara \& Balluerka, 2000). Luego de haber completado el proceso de traducción y valoración de la comprensión de los ítems, se procedió a conducir el estudio en sí. Se invitó a los participantes a través de distintos medios, tales como redes sociales (Facebook), correos electrónicos y carteles en las facultades. Los participantes recibieron información sobre las condiciones del estudio y brindaron su consentimiento informado.

Los participantes informaron diariamente si experimentaron envidia a lo largo de 15 días consecutivos, considerando el primer día como día de prueba. Recibieron todos los días un correo electrónico a las 17 hs. con un link para completar la información correspondiente a ese día. Si experimentaban envidia, debían describir la experiencia de envidia y responder una serie de preguntas respecto a la intensidad, facilidad, contenido experiencial y sobre aspectos valorativos. Además, el primer día del estudio, los participantes respondieron a escalas para medir variables disposicionales. El cuestionario de cada día estuvo habilitado desde las 19hs. hasta las OOhs. Luego de completar la información correspondiente a los días 4, 7, 10, 12 y 14, los participantes que respondieron esos días, recibieron un número para participar en un sorteo por una entrada al cine. Los participantes recibieron información el mismo día sobre los resultados del sorteo para incentivarlos a continuar con la tarea. Al finalizar el estudio, todos los participantes recibieron nuevos números de acuerdo a la cantidad de días que respondieron durante el estudio y se realizó un nuevo sorteo de dos órdenes de compra de $\$ 150$ cada una para libros y/o música, los números ganadores se determinaron según sorteo de organismos oficiales de lotería.

\section{Análisis de datos}

Inicialmente se realizaron una serie de análisis descriptivos. Luego se desarrolló un análisis de clases latentes (Hagenaars \& McCutcheon, 2002; Reyna \& Brussino, 2011) para identificar los singulares patrones de respuestas (contenido experiencial) asociados a ambos tipos de envidia. Después se utilizó la prueba $U$ de Mann-Whitney para evaluar las diferencias en aspectos valorativos según la experiencia de envidia benigna o maligna (Aron \& Aron, 2001). Por último, se realizaron análisis de diferencias de medias (pruebas $t$ para muestras independientes) para determinar si los participantes que experimentaron envidia benigna y maligna presentaron diferentes niveles promedio de envidia disposicional y derecho psicológico (entitlement). Se estableció un nivel de significación de 0.05 . Estos análisis fueron conducidos con los programas estadísticos Latent Gold 4.0 y SPSS 19.

\section{Aspectos éticos}

Se respetaron los lineamientos éticos para la investigación con humanos recomendados por la Asociación Psicológica Americana (Ethical principles of psychologist and code of conduct, Washington, DC, American Psychological Association, 2010). Los investigadores involucrados en el presente proyecto consideramos las salvaguardas previstas en las normas bioéticas nacionales Disposición 
ANMAT (1997) 5330/97 e internacionales (Declaración de Helsinski y sus modificaciones).

\section{Resultados}

De la muestra total de participantes, 33 $(61.11 \%)$ indicaron haber experimentado envidia durante el período del estudio, 28 de sexo femenino $(84.8 \%)$ y 5 de sexo masculino (15.2\%), con una media de edad de 21.39 años $(\mathrm{DS}=1.94)$. Los participantes que experimentaron envidia, lo hicieron en promedio después de 3.30 días $(\mathrm{DS}=2.8)$ de haber comenzado el estudio, experimentando envidia un total de dos días en promedio ( $\mathrm{DS}=$ 1.17). En total, se reportaron 66 experiencias de envidia durante los 14 días de estudio. Además, se observó que el nivel de intensidad de la experiencia de envidia fue medio $(M=4.09, D S=2.16)$. En tanto que fue relativamente fácil recordar las experiencias de envidia que reportaron $(\mathrm{M}=6.03, \mathrm{DS}=2.48)$.

Se desarrolló un análisis de clases latentes a partir de los indicadores sobre contenido experiencial para evaluar el contenido experiencial asociado a la envidia maligna y benigna. Concretamente, el análisis se condujo en base a los ítems de la primera experiencia de envidia que los participantes experimentaron durante el estudio, al igual que en otros estudios (Van de Ven et al., 2009). Se desarrollaron modelos de 1 a 3 clases latentes. Se evaluó el valor de L2 y se compararon los modelos en las medidas que tienen en cuenta tanto la bondad del ajuste como la parsimonia: BIC, AIC, AIC3 (basándose en LL).

El análisis indicó que el modelo de 2 clases latentes fue el que presentaba un mejor ajuste. Para este modelo se observó que el BIC, el AIC, y el AIC3 tenían el valor más bajo (433.5, 399.08 y 422.08 , respectivamente). Además, se consideró el error de clasificación, el cual fue de $4.84 \%$ para el modelo de 2 clases. En la Tabla 1 se detallan los valores de los índices de bondad de ajuste de los modelos de 1 a 3 clases latentes.
TABLA 1

Índices de bondad de ajuste de los modelos de 1 a 3 clases latentes

\begin{tabular}{llllllll}
\hline & LL & BIC(LL) & AIC(LL) & AIC3(LL) & L2 & $P$ & $\begin{array}{l}\text { Error de } \\
\text { Clasificación }\end{array}$ \\
\hline 1-Cluster & -195.56 & 440.06 & 419.11 & 433.11 & 168.66 & $5.2 \mathrm{E}-26$ & 0 \\
2-Cluster & -176.54 & 433.5 & 399.08 & 422.08 & 130.63 & $3.5 \mathrm{E}-23$ & 0.0484 \\
3-Cluster & -171.37 & 454.63 & 406.75 & 438.75 & 120.3 & $5.4 \mathrm{E}-28$ & 0.0889 \\
\hline
\end{tabular}

Fuente: elaboración propia.

También se valoraron los efectos asociados a cada una de las variables indicadoras a través del estadístico Wald. Se consideró un valor $\mathrm{p}=$ 0.05 , por lo que un valor $\mathrm{p}<0.05$ significa que conocer la respuesta a ese indicador contribuye a discriminar entre las clases. Así, los indicadores "me sentí inspirado/a por la persona a la que envidié" ( $\mathrm{p}=0.042)$, "felicité a la otra persona por su éxito" $(\mathrm{p}=0.014)$, "me sentí distante de la persona a la que envidié" $(\mathrm{p}=0.035)$ y "lo sentí frustrante" $(p=0.014)$ resultaron ser significativos para diferenciar las clases (Tabla 3). Mientras que los indicadores lo "sentí placentero", "me esforcé para lograr mis objetivos", "esperé que le fuera mal a la persona que envidié" y "me quejé con otros sobre la persona que envidié" no fueron significativos.

Tal como se esperaba, el análisis de clases latentes contribuyó a identificar los singulares patrones de respuestas (contenido experiencial) asociados a ambos tipos de envidia, observando en los resultados que las diferencias en las medias de las dos clases se ajustan a la distinción entre la envidia maligna y benigna (Figura 1 y Tabla 2). Los participantes que experimentaron envidia maligna se sintieron más distantes de la persona a las que envidiaban y, además, sintieron dicha experiencia como más frustrante que las personas que experimentaron envidia benigna. Mientras que los participantes que experimentaron envidia benigna se sintieron más inspirados por la persona a la que envidiaron y felicitaron más a la otra persona por su éxito que las personas que experimentaron envidia maligna. De los 33 participantes, 12 (36\%) informaron haber experimentado envidia benigna, en tanto que el resto de los participantes experimentaron envidia maligna. 


\section{Figura 1}

Probabilidad estimada de las experiencias de envidia maligna y benigna

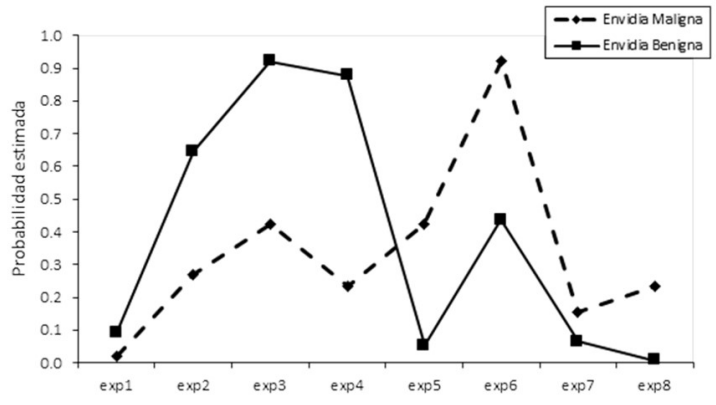

exp1 = lo sentí placentero, exp2 = me sentí inspirado/a por la persona a la que envidié, exp3= me esforcé más para lograr mis objetivos, exp4= felicité a la otra persona por su éxito, $\exp 5=$ me sentí distante de la persona a la que envidié, $\exp 6=$ lo sentí frustrante, $\exp 7=$ esperé que le fuera mal a la persona que envidié, $\exp 8=$ me quejé con otros sobre la persona a la que envidié. Fuente: elaboración propia.

TABLA 2

Resultados del análisis de clases latentes del contenido experiencial de envidia

\begin{tabular}{|c|c|c|c|c|c|}
\hline \multirow[b]{2}{*}{ Cuando experimenté envidia... } & \multicolumn{2}{|l|}{$M(D S)$} & \multicolumn{3}{|c|}{ Efecto de los indicadores } \\
\hline & $\begin{array}{l}\text { Clase 1: Envidia } \\
\text { maligna }\end{array}$ & $\begin{array}{l}\text { Clase 2: Envidia } \\
\text { benigna }\end{array}$ & Wald & $p$ & $r^{2}$ \\
\hline Lo senti placentero. & $1.04(0.06)$ & $1.18(0.16)$ & 0.67 & 0.41 & 0.033 \\
\hline $\begin{array}{l}\text { Me sentí inspirado/a por la persona a la que } \\
\text { envidié. }\end{array}$ & $1.54(0.19)$ & $2.29(0.26)$ & 4.15 & 0.042 & 0.16 \\
\hline Me esforcé más para lograr mis objetivos. & $1.84(0.21)$ & $2.84(0.19)$ & 2.8 & 0.094 & 0.3 \\
\hline Felicité a la otra persona por su éxito. & $1.46(0.18)$ & $2.75(0.19)$ & 6.07 & 0.014 & 0.49 \\
\hline Me sentí distante de la persona a la que envidié. & $1.85(0.15)$ & $1.11(0.12)$ & 4.43 & 0.035 & 0.3 \\
\hline Lo sentí frustrante. & $2.84(0.1)$ & $1.87(0.3)$ & 6.07 & 0.014 & 0.36 \\
\hline Esperé que le fuera mal a la persona que envidié. & $1.15(0.08)$ & $1.06(0.09)$ & 0.34 & 0.56 & 0.018 \\
\hline $\begin{array}{l}\text { Me qujé con orros sobre la persona a la que } \\
\text { envidie. }\end{array}$ & $1.47(0.19)$ & $1.01(0.05)$ & 1.05 & 0.31 & 0.095 \\
\hline$n$ & 21 & 12 & & & \\
\hline
\end{tabular}

Fuente: elaboración propia.

Se condujeron análisis de comparación de grupos para analizar si los participantes que experimentaron envidia benigna y maligna presentaban puntuaciones diferentes en las dimensiones sobre aspectos valorativos. Dado que estas dimensiones presentaban distribuciones no normales y varianzas no homogéneas en cada una de las clases latentes, se utilizaron pruebas no paramétricas (Aron \& Aron, 2001). Específicamente, se recurrió a la prueba $U$ de Mann-Whitney.

El análisis de comparación indicó una diferencia estadísticamente significativa entre las clases latentes para las dimensiones "estado situacional" $(\mathrm{U}=30, \mathrm{p}=0, \mathrm{r}=-0.65)$, "poder propio" $(\mathrm{U}=48.5, \mathrm{p}=0.003, \mathrm{r}=-0.51)$ y "merecimiento" $(U=75, p=0.047, r=$ -0.35). Esto es, las personas que sintieron envidia benigna creyeron que la situación mejoraría las cosas en mayor medida que las personas que sintieron envidia maligna. Además las personas que experimentaron envidia benigna sintieron que tenían mayor poder sobre la situación que las personas que experimentaron envidia maligna. Asimismo, las personas que sintieron envidia benigna valoraron la situación como más merecida que las personas que sintieron envidia maligna (Tabla 3). Por otro lado, el análisis de comparación evidenció resultados no significativos para las dimensiones "no esperado", "estado motivacional", "probabilidad", "legitimidad" y "agencia".

\section{TABLA 3}

Resultados del análisis de comparación para las dimensiones de los aspectos valorativos de envidia maligna y benigna

\begin{tabular}{|c|c|c|c|c|}
\hline \multirow[b]{2}{*}{ Dimensión } & \multicolumn{2}{|l|}{ Rango promedio } & \multirow[b]{2}{*}{$U$} & \multirow[b]{2}{*}{$P$} \\
\hline & $\begin{array}{l}\text { Clase 1: Envidia } \\
\text { maligna }\end{array}$ & $\begin{array}{l}\text { Clase 2: Envidia } \\
\text { benigna }\end{array}$ & & \\
\hline No esperado & 17 & 17 & 126 & 1 \\
\hline Estado de la situación & 12.43 & 25 & 30 & 0 \\
\hline Estado motivacional & 14.9 & 20.67 & 82 & 0.095 \\
\hline Probabilidad & 15.67 & 19.33 & 98 & 0.283 \\
\hline Legitimidad & 15.26 & 20.04 & 89.5 & 0.167 \\
\hline Poder propio & 13.31 & 23.46 & 48.5 & 0.003 \\
\hline Agencia-yo & 17.93 & 15.38 & 106.5 & 0.46 \\
\hline Agencia-otro & 15.19 & 20.17 & 88 & 0.143 \\
\hline Agencia-circunstancias & 16.62 & 17.67 & 118 & 0.76 \\
\hline Merecimiento & 14.57 & 21.25 & 75 & 0.047 \\
\hline
\end{tabular}

Nota. p: significación asintótica bilateral.

Fuente: elaboración propia.

A continuación, se compararon los niveles de envidia disposicional y derecho psicológico (entitlement) entre los participantes de las dos clases latentes, para lo cual se condujeron pruebas t para muestras independientes (Aron \& Aron, 2001).

Primero se consideró la variable envidia disposicional. Dado que los análisis de distribución y homogeneidad de varianzas indicaron que la variable presentaba una distribución normal y homogénea en cada una de las clases latentes, se procedió con el análisis paramétrico. El análisis de comparación de medias de envidia disposicional indicó una diferencia estadísticamente significativa entre las 
clases latentes $(\mathrm{t}(31)=2.66, \mathrm{p}=0.01, \mathrm{~d}=$ 0.13). Las personas que sintieron envidia maligna $(\mathrm{M}=2.4, \mathrm{DS}=0.63)$ experimentaron un mayor nivel de envidia disposicional que las personas que sintieron envidia benigna $(\mathrm{M}=1.82 ; \mathrm{DS}=0.53)$.

Posteriormente, se analizaron los resultados para la variable derecho psicológico (PES). Los análisis de distribución y de homogeneidad de varianzas indicaron que la variable presentaba una distribución normal y homogénea en cada una de las clases latentes, por lo tanto, se utilizó el análisis paramétrico. El análisis de comparación de medias para la variable derecho psicológico (entitlement) no mostró diferencias estadísticamente significativas entre las clases latentes ( $\mathrm{t}(31)=0.04, \mathrm{p}=0.97)$. Además, se calculó la prueba $t$ excluyendo del análisis el caso atípico, y se obtuvieron resultados semejantes. Tomados en conjunto, estos resultados indican que las personas que experimentaron envidia maligna manifestaron un mayor nivel de envidia disposicional que las personas que experimentaron envidia benigna, y que no existe diferencia en los niveles de derecho psicológico (entitlement) entre los participantes que experimentaron envidia benigna y maligna.

\section{Discusión}

Este trabajo se propuso evaluar el contenido experiencial y el patrón valorativo de la envidia benigna y de la envidia maligna en estudiantes universitarios de Córdoba, Argentina. Los resultados obtenidos permitieron abordar cuestiones teóricas de la envidia benigna y maligna en el contexto local. A continuación, se sintetizan y discuten los resultados obtenidos en el marco de los estudios actuales sobre envidia, se plantean las limitaciones de este trabajo y se proponen futuras líneas de investigación.

Tradicionalmente las investigaciones se focalizaron en el estudio de la envidia como una emoción homogénea, desagradable, que se caracteriza por sentimientos de inferioridad, de mala voluntad y de injusticia (Smith et al., 1999). Sin embargo, trabajos recientes, llevadds Universitas Psychologica | V. i6 | No. 2 | AbriL-Junio | 2017 |

a cabo en diferentes países, observaron la existencia de una forma de envidia cualitativamente distinta. Es decir, un tipo de envidia más positiva que produce motivación para mejorar y que ha sido denominada en la literatura científica como envidia benigna (e.g., Lange \& Crusius, 2015; Van de Ven et al., 2009). Acorde con los resultados encontrados en otros países, este estudio evidenció que en el contexto local las personas describieron diferentes tipos de envidia (maligna y benigna) cuando reportaron haber experimentado esta emoción. En otras palabras, los dos tipos de envidia se caracterizaron por poseer distintos contenidos experienciales y aspectos valorativos.

Con respecto al contenido experiencial, cuando los participantes cordobeses experimentaron envidia maligna se sintieron más distantes de la persona a las que envidiaban y, además, sintieron dicha experiencia como más frustrante que cuando experimentaron envidia benigna. Mientras que los participantes que experimentaron envidia benigna se sintieron más inspirados por la persona a la que envidiaron y felicitaron más a la otra persona por su éxito que cuando experimentaron envidia maligna. En cuanto a los aspectos valorativos, las personas que sintieron envidia benigna creyeron que la situación mejoraría las cosas, sintieron que tenían mayor poder sobre la situación y valoraron dicha situación como más merecida que las personas que sintieron envidia maligna. Incluso, considerando el porcentaje de participantes que (36\%), España (38\%) y Estados Unidos (54\%) o forma de la envidia que, tal como se explicitó anteriormente, ha sido ignorada en la investigación durante un largo tiempo (Van de Ven et al., 2009).

A pesar de que la envidia ha sido considerada una emoción universal, los resultados del presente estudio evidencian ciertas particularidades de la experiencia de sentir envidia benigna y maligna en nuestro contexto. Concretamente, en estudios con muestras de estadounidenses y españolas, las personas que experimentaron envidia benigna sentían la situación más placentera y se esforzaban para experimentaron envidia benigna en Argentina vale señalar que es una importante faceta 
lograr sus objetivos, mientras que las personas que sintieron envidia maligna esperaban que les fuera mal y se quejaban con otros sobre la persona envidiada, además de puntuar alto en el resto de los ítems utilizados para medir el contenido experiencial (Van de Ven et al., 2009). A su vez, las personas que sintieron envidia benigna valoraron la situación como más controlable, a diferencia de lo evidenciado en la muestra local. En consecuencia, es posible considerar que las diferencias observadas entre nuestro estudio y los de Van de Ven et al. (2009; 2012) se deban a variaciones a nivel individual o cultural en la experiencia de envidia (Fischer \& Manstead, 2008), sobre todo al considerar que la envidia es una emoción social que surge en un contexto social específico (Chóliz \& Gómez, 2002).

En cuanto a la evaluación de las diferencias en la envidia disposicional (DES) y el derecho psicológico (PES) se observó, por un lado, que los participantes que sintieron envidia maligna experimentaron un mayor nivel de envidia disposicional que los participantes que sintieron envidia benigna (grupos derivados del análisis de clases latentes), en concordancia con lo postulado por Smith et al. (1999) y Smith y Kim (2007). Por otro lado, no se encontraron diferencias en los niveles de derecho psicológico (entitlement) entre los participantes que experimentaron envidia maligna y envidia benigna, coincidente con los antecedentes teóricos que distinguen el derecho psicológico (entitlement) del merecimiento (deservingness) (Feather, 2003; Van de Ven et al., 2012). Es decir, el derecho psicológico no permite diferenciar entre las personas envidiosas que se consideran merecedoras de la situación y aquellas que no.

$\mathrm{Si}$ bien se logró responder al objetivo propuesto en el presente trabajo, es necesario señalar algunas limitaciones. Se utilizó un muestreo no probabilístico, lo cual limita la generalización de los resultados a las poblaciones de referencia. El uso del muestreo auto-elegido se debió a la accesibilidad y disponibilidad de recursos humanos para la recolección de datos. Asimismo, es posible que la deseabilidad social haya influido sobre las respuestas de los participantes, aspecto que no fue controlado en este estudio. No obstante, los instrumentos de medición fueron auto-administrados y aplicados de manera individual sin la presencia del investigador. A su vez, respecto a la evaluación de la respuesta emocional vale señalar que se realizó mediante instrumentos de auto-reporte que aportan información únicamente sobre los aspectos conscientes de la respuesta emocional (Grygolec, Coricelli, \& Rustichini, 2009).

Futuros trabajos podrían avanzar en la implementación de vías de medición emocional diferentes a los instrumentos de auto-reporte. Esto es, algunos autores sostienen que para mejorar la validez, las emociones podrían ser medidas mediante la respuesta galvánica de la piel, el ritmo cardíaco, la actividad bioeléctrica cerebral (EEG), cardiovascular, respiratoria y mioeléctrica gástrica, entre otras (Bosman, Sonnemans, \& Zeelenberg, 2001; Tranel, 2006). También se podrían estudiar otras emociones relacionadas con la envidia maligna y benigna, por ejemplo, el resentimiento y la admiración, y así identificar las particularidades de cada experiencia emocional en el contexto local. Si bien es cierto que estas emociones pueden ser desencadenadas en situaciones de comparación social ascendente, se caracterizan por poseer contenidos experienciales y patrones valorativos específicos que pueden originar comportamientos diferentes (Van de Ven et al., 2012).

Adicionalmente, considerando que la envidia es una emoción social que puede provocar respuestas cooperativas o egoístas (Pfister \& Böhm, 2012) y teniendo en cuenta los aportes recientes sobre el rol de la envidia en la toma de decisiones, sería positivo estudiar el rol mediacional de la envidia benigna y maligna en situaciones de interacción estratégica, es decir, en situaciones en las cuales la decisión individual se ve influenciada por las decisiones de los otros. A su vez, considerando que estudios previos han señalado los efectos de la envidia sobre el desempeño grupal (Duffy \& Shaw, 2000; Kim, O’Neill \& Cho, 2010), sería positivo indagar el rol de la envidia benigna sobre la cohesión grupal, dado que dicha emoción podría desencadenar, por un lado, un comportamiento positivo para 
el grupo o, por otro lado, un

comportamiento potencialmente dañino.

En síntesis, se destaca que los resultados observados en esta investigación han aportado evidencia sobre diferencias en el contenido experiencial y los aspectos valorativos de la envidia benigna y maligna en muestras de estudiantes universitarios de Córdoba, Argentina.

\section{Agradecimientos}

Institución que financió la investigación: Secretaría de Ciencia y Tecnología Universidad Nacional de Córdoba (Proyecto "Toma de decisiones económicas y procesos emocionales: análisis Instrumental y experimentos en situaciones de consumo individual e interpersonal", Directora: Cecilia Reyna).

\section{Referencias}

American Psychological Association (2010).

Ethical principles of

psychdlogístiscallakthihngtertigación en Estudios de ANMFAitmac(19g/7)ClínRégimieposicalén 5330/97, Buenßsuenos Aires.

Aron, A., \& Aron, E. N. (2001). Estadística para psicología. Buenos Aires: Pearson Educación.

Bosman, R., Sonnemans, J., \& Zeelenberg, M. (2001). Emotions, rejections, and cooling off in the ultimatum game. Unpublished manuscript, University of Amsterdam.

Campbell, W. K., Bonacci, A. M., Shelton, J., Exline, J. J., \& Bushman, B. J. (2004). Psychological entitlement: interpersonal consequences and validation of a self-report measure. Journal of Personality Assessment, 83(1), 29-45. doi: http://dx.doi.org/10.120 7/s15327752jpa8301_04

Chóliz, M., \& Gómez, C. (2002). Emociones sociales II (enamoramiento, celos, envidia y empatía). En F. Palmero, E.G. FernándezAbascal, F. Martinez y M. Chóliz (Eds.),
Psicología de la Motivación y Emoción (pp. 395-418). Madrid: McGraw Hill.

Cohen-Charash, Y. (2009). Episodic envy. Journal of Applied Social Psychology, 39(9), 2128 2173. doi: http://dx.doi.org/10.1111/j.1559 $-1816.2009 .00519 . x$

Crusius, J., \& Lange, J. (2014). What catches the envious eye? Attentional biases within malicious and benign envy. Journal of Experimental Social Psychology, 55, 1-11. doi: http://dx.doi.org/10.1016/j.jesp.2014.0 5.007

Duffy, M. K., \& Shaw, J. D. (2000). The Salieri syndrome: Consequences of envy in groups. Small Group Research, 31, 3-23. doi: http:// dx.doi.org/10.1177/104649640003100101

Dvash, J., Gilam, G., Ben-Ze'ev, A., Hendler, T., \& Shamay-Tsoory, S. G. (2010). The envious brain: the neural basis of social comparison. Human Brain Mapping, 31 (11), 1741-1750. doi: http://dx.doi.org/10.1002/ hbm.20972

Feather, N. T. (2003). Distinguishing between deservingness and entitlement: earned outcomes versus lawful outcomes. European Journal of Social Psychology, 33(3), 367-385. doi: http://dx.doi.org/10.1002/ejsp.152

Feather, N. T. (2006). Deservingness and emotions: applying the structural model of deservingness to the analysis of affective reactions to outcomes. European Review of Social Psychology, 17(1), 38-73. doi:10.1080/10463280600662321

Feather, N. T., \& McKee, I. R. (2009). Differentiating emotions in relation to deserved undeserved outcomes: a retrospective study of real-life events. Cognition and Emotion, 23(5), 955-977. doi: http://dx.doi.org/10.1080/0269993080 2243378

Fischer, A. H., \& Manstead, A. S. R. (2008). Social functions of emotions. In M, Lewis, J. M. Haviland-Jones \& L. Feldman Barret (Eds.), Handbook of Emotions (pp. 456-465). New York: The Guildford Press.

Foster, G. M. (1972). The anatomy of envy: a study in symbolic behavior. 
Current Anthropology, 13(2), 165-202. doi: $10.1086 / 201267$

Grygolec, J., Coricelli, G., \& Rustichini, A. (2009). Neuroeconomics of 3-person ultimatum game with Voting: the case of responders. Paper session presented at the meeting of the Faculty of Graduate School of University of Minnesota, USA.

Hagenaars, J. A., \& McCutcheon, A. L. (2002). Applied latent class analysis. New York: Cambridge University Press.

Hoelzl, E., \& Loewenstein, G. (2005).Wearing out your shoes to prevent someone else from stepping into them: anticipated regret and social takeover in sequential decisions. Organizational Behavior and Human Decision Processes, 98(1), 15-27. doi: http://dx.doi.o rg/10.1016/j.obhdp.2005.04.004

Jankowski, K. F., \& Takahashi, H. (2014). Cognitive neuroscience of social emotions and implications for psychopathology: examining embarrassment, guilt, envy, and schadenfreude. Psychiatry and Clinical Neurosciences, 68(5), 319-336. doi: http://d x.doi.org/10.1111/pcn.12182

Kahneman, D., Krueger, A. B., Schkade, D. A., Schwarz, N., \& Stone, A. A. (2004). A survey method for characterizing daily life experience: the day reconstruction method. Science, 306(5702), 1776-1780. doi: http:// dx.doi.org/10.1126/science.1103572

Kim, S., O’Neill, J. W., \& Cho, H. M. (2010). When does an employee not help coworkers? The effect of leader-member exchange on employee envy and organizational citizenship behavior. International Journal of Hospitality Management, 29(3),530-537. doi: http://dx .doi.org/10.1016/j.ijhm.2009.08.003

Lange, J., \& Crusius, J. (2015). Dispositional envy revisited: unraveling the motivational dynamics of benign and malicious envy. Personality and Social Psychology Bulletin, 41 (2), 284-294. doi: http://dx.doi.org/10.11 77/0146167214564959

Mola, D. J., Saavedra, B. A., Reyna, C. E., \& Belaus, A. (2013). Valoración psicométrica de la Psychological Entitlement Scale desde la Teoría Clásica de los Test y la Teoría de Respuesta al Ítem. Pensamiento Psicológico, 11(2), 19 -38.

Mola, D. J., Saavedra, B. A., \& Reyna, C. E. (2014). Evidences of reliability and validity of the Dispositional Envy Scale. International Journal of Psychological Research, 7(1), 73-80.

Mola, D. J., Reyna, C. E., \& Godoy, J. C. (2015). El rol de la envidia benigna y maligna en la toma de decisiones estratégicas. Suma Psicológica, 22(1), 53-61.

Parks, C. D., Rumble, A. C., \& Posey, D. C. (2002). The effects of envy on reciprocation in a social dilemma. Personality and Social Psychology Bulletin, 28(4), 509-520. doi: htt p://dx.doi.org/10.1177/0146167202287008

Parrott, W. G., \& Smith, R. H. (1993). Distinguishing the experiences of envy and jealousy. Journal of Personality and Social Psychology, 64(6), 906-920. doi: http://dx.d oi.org/10.1037/0022-3514.64.6.906

Pfister, H. R., \& Böhm G. (2012). Responder feelings in a three-player three-option ultimatum game: Affective determinants of rejection behavior. Games, 3, 1-29. doi: htt p://dx.doi.org/10.3390/g3010001

Reyna, C., \& Brussino, S. (2011). Revisión de los fundamentos del análisis de clases latentes y ejemplo de aplicación en el área de las adicciones. Trastornos Adictivos, 13(1), 11-19.

Roseman, I. J., Antoniou, A. A., \& Jose, P. E. (1996). Appraisal determinants of emotions: Constructing a more accurate and comprehensive theory. Cognition $\mathbb{E}$ Emotion, 10(3), 241-277. doi: http://dx.doi. org/10.1080/026999396380240

Roseman, I. J., Wiest, C., \& Swartz, T. S. (1994). Phenomenology, behaviors, and goals differentiate discrete emotions. Journal of Personality and Social Psychology, 67(2), 206-221. doi: http://dx.doi.org/10.10 37/0022-3514.67.2.206

Shamay-Tsoory, S. G., Tibi-Elhanany, Y., \& Aharon-Peretz, J. (2007). The greeneyed monster and malicious joy: the neuroanatomical bases of envy and 
gloating (schadenfreude). Brain, 130

(6), 1663-1678.

Smith, R. H., Parrot, W. G., Diener, E. F., Hoyle R. H., \& Kim, S. H. (1999). Dispositional envy. Personality and Social Psychology Bulletin, 25 (8), 1007-1020. doi: http://dx.d oi.org/10.1037/0022-3514.67.2.206

Smith, R. H., \& Kim, S. H. (2007). Comprehending envy. Psychological Bulletin, 133(1), 46-64. doi: http://dx.doi.org/10.103 7/0033-2909.133.1.46

Sterba, S. K., \& Foster, E. M. (2008).Selfselected sample. In P. J. Lavrakas (Ed.), Encyclopedia of Survey Research Methods (pp. 806-808). Thousand Oaks, California: SAGE Publications.

Tranel, D. (2006). Preface: Psychophysiology and cognitive neuroscience. International Journal of Psychophysiology, 61 (1), 1-4.

Van de Ven, N, (2009). The bright side of a deadly sin the psychology of envy. Doctoral thesis, University of Tilburg, Netherlands.

Van de Ven, N., Zeelenberg, M., \& Pieters, R. (2009). Leveling up and down: the experiences of benign and malicious envy. Emotion, 9(3), 419-429. doi: http://dx.doi.o $\mathrm{rg} / 10.1037 / \mathrm{a} 0015669$

Van de Ven, N., Zeelenberg, M., \& Pieters, R. (2010). Warding off the evil eye: when the fear of being envied increases prosocial behavior. Psychological Science, 21 (11), 1671-1677. doi: http://dx.doi.org/1 $0.1177 / 0956797610385352$

Van de Ven, N., Zeelenberg, M., \& Pieters, R. (2012). Appraisal patterns of envy and related emotions. Motivation and Emotion, 36(2), 195-205.

Vergara, A. I., \& Balluerka, N. (2000). Metodología en la investigación transcultural: perspectivas actuales. Psicothema, 12 (2), 557-562.

Zeelenberg, M., \& Pieters, R. (2006). Feeling is for doing: a pragmatic approach to the study of emotions in economic behavior. In D. De Cremer, M. Zeelenberg, \& K. Murnighan (Eds.), Social Psychology and Economics (pp. 117-137). Mahwah, NJ: Erlbaum.

\section{Notas}

* Artículo de investigación 